\title{
Experimental Investigations of Force Character of Extra Vehicular Activity Glove in Different Pressure Levels
}

\author{
Hanjun YANG ${ }^{a}$, Chunhui WANG ${ }^{b}$, Peng TENG, Li WANG, Shihua ZHOU \\ Science and Technology on Human Factors Engineering Laboratory \\ China Astronaut Research \& Training Center \\ Beijing, China \\ ayanghj1886@126.com, bchunhui_89@yahoo.com.cn
}

Keywords: ergonomics experiment; space suit; EVA glove; correlation analysis; pressure level.

\begin{abstract}
This article researches the force characters of the Extra Vehicular Activity (EVA) glove under different pressure conditions using an ergonomic experimental method. First, 20 male subjects are selected to operate a series of operation actions with their bare hands. Some typical operation actions, such as hold, screw and pinch, are requested to collect the force data of these subjects. Second, these subjects are asked to repeat these operation actions above again when they wear the EVA glove under the different pressure level. Finally, we use some correlation analysis techniques to mine the relationship among the collected data of different pressure levels and different wearing states. Experiment results show us: the force data of bare hand state have a correlation with the force data of wearing EVA glove state. So, this result can be use to analyze and estimate the force size of the wearing EVA glove state when the astronauts work in the space station.
\end{abstract}

\section{Introduction}

With the increased number of scientific research tasks in orbit, the Extra Vehicular Activity (EVA) [1] calls for a higher technique requirement to the space suit of China. The EVA glove is one of most important module of the space suit for the astronauts when they execute some missions out of the space station. The basic functions of the EVA glove [2] include not only to protect the hands of the astronaut from the extreme hard space environment but to assist the astronaut to finish some complex operation tasks flexibly. Fig. 1 shows us the EVA glove of space suit. In Fig. 1 the EVA glove include at least the atmoseal layer, the framework layer and the thermal micrometeoroid garment layer. So the complex structure of the glove will affect its usability seriously.

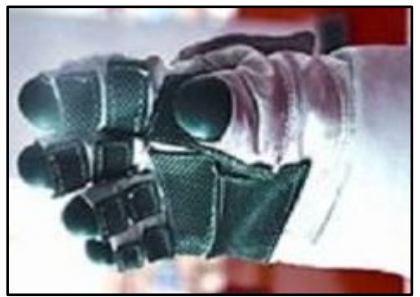

(a)

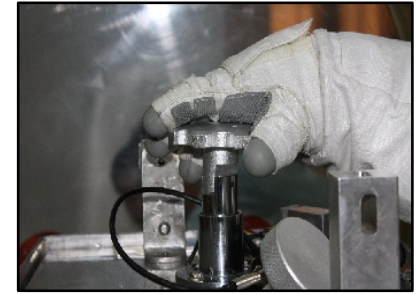

(b)

Figure 1. The EVA glove of a tpye of space suit.

Many research

to evaluate the usability of the EVA glove. In [3], the authors studied the force endurance capabilities of the EVA glove in different pressure levels. The authors analyzed the endurance time and concluded that the performance decrement would happen with the pressure increasing of the glove. In [4] the authors evaluated the performance of the hybrid elastic EVA glove. The authors compared the flexibility between the hybrid elastic EVA glove and the 4000-series EMU glove to evaluate the function of it. In [5], the authors used the experimental method to get quantified data results of the finger tactility of their EVA glove. As for our EVA glove, on one hand, the design and production of the EVA glove is different to the version of foreign one. On the other hand, with the development of 
the space technique in China, we also need to know the force rule of the EVA glove. So it is necessary to research the force character of the EVA glove from the ergonomic research point of view.

In this paper, an experimental investigation is applied to research the force character of the EVA glove under different pressure level. First, we test the basic force character of 20 male subjects. Some typical operation actions, such as hold, screw and pinch, are arranged when the subjects use their bare hands to finish the appointed operation missions. Second, we ask the subjects to wear the EVA glove to finish the same tasks under different pressure level. The pressure level of $0 \mathrm{kPa}$ and the level of $40 \mathrm{kPa}$ are arranged to collect the force character data of these ergonomic experiments. Third, we employ the correlation analysis method and the regression analysis method to mine the change rules among the force data of different situations. Finally, the experiment analysis results show us the force data of bare hand have a correlation with the data of wearing EVA glove state under $40 \mathrm{kPa}$ pressure level indeed.

The main contributions of this paper include: 1) we study the typical operation force characters of a certain type of EVA glove of the space suit. 2) We proof the existence of the correlation between the typical force data of bare hand and that of the wearing EVA glove state quantificationally.

\section{Methodologies}

Subjects. 24 male subjects are selected to finish this typical force measurement experiment. All the subjects are right handed, from 25 to 45 years old. Their heights are from $165 \mathrm{~cm}$ to $175 \mathrm{~cm}$. Their weights are from $65 \mathrm{~kg}$ to $75 \mathrm{~kg}$. The Body Mass Index (BMI) of them is from 19 to 25. The girth of their forearm does not large than $29 \mathrm{~cm}$. After some simple training they are familiar with all the operation experiment steps of the EVA glove.

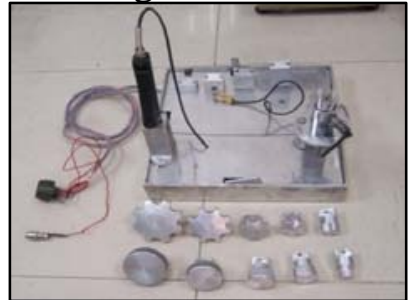

(a)

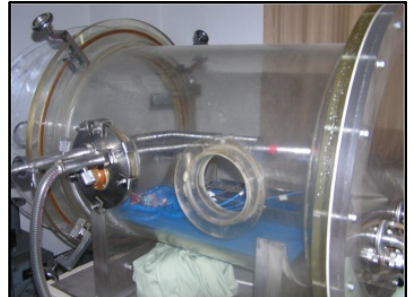

(b)

Figure 2 The corresponding force measurement device.

\section{Experimental}

Devices. All

the experiment devices are shown in Fig. 1 and Fig. 2. The experimental devices include: the EVA glove, the negative pressure cabin, the force sensor and the data record and display terminal. First, the EVA glove has a similar function like any ordinary gloves. The finger part of the EVA glove can be crooked. The wrest part of it can be twisted. However, since the EVA glove will work in some inclement space environments, the structure of it is very complex. As a result, its ergonomic efficiency will decrease $80 \% \sim 90 \%$ than that of the bare hand situation. Second, the negative pressure cabin can be used to control the pressure of this experiment. By tuning the adjustment device, its pressure can reach $90 \mathrm{kPa}$ to $100 \mathrm{kPa}$. The negative pressure cabin also provides installation space for the EVA glove and corresponding electronical and mechanical interface. Third, the force sensor can measure the typical operation force of hold, screw and pinch in real time. Fourth, the data record and display terminal will record the measurement results and lay out the data result in the form of multimedia mode.

Procedures. 1) The basic force measurement experiment. The experiment steps include: 1) the experimenter test all the states of the experiment devices. 2) The subject sits in front of the negative pressure cabin and uses the EVA glove to finish the typical operation tasks. The force sensors and corresponding tools will measure the typical force of hold, screw and pinch respectively. The force moment parameters of the measurement device are shown in Table 1. 3) The experimenter monitors the record results of all these experiment data and saves them correctly. 4) When each of the force measurement experiment is over, the subject can have a rest until his fatigue disappears completely. 5) Each experiment will be repeated for 3 times. 6) The subjects are asked to repeat the same experiment 
under three different situations: the state of bare hand, the state of wearing EVA glove under 0kPa pressure and the state of wearing EVA glove under 40kPa pressure respectively.

Table1 Force Momet Parameters of Different Force Measurement Devices.

\begin{tabular}{|c|c|}
\hline Measurement Index & Size of Force Moment \\
\hline Hold Force & $40 \mathrm{~mm}, 50 \mathrm{~mm}, 60 \mathrm{~mm}, 70 \mathrm{~mm}$ \\
\hline Screw Force & $15 \mathrm{~mm}, 25 \mathrm{~mm}, 40 \mathrm{~mm}, 55 \mathrm{~mm}, 70 \mathrm{~mm}$ \\
\hline Pinch Force & $20 \mathrm{~mm}, 40 \mathrm{~mm}, 55 \mathrm{~mm}, 70 \mathrm{~mm}$ \\
\hline
\end{tabular}

2) The integrated force measurement experiment. Different to the basic experiment, the integrated experiment needs the subject to finish a complex operation task: he is asked to screw a nut on a bolt (see Fig. 2) under different wearing states and different pressure levels. In this experiment, the spec of the nut and bolt is 4 . When the experiment begins, the subject has to select and assembles the right nut on the right bolt as quick as possible. The experimenter will record the operation time of each experiment. Each experiment will be repeated for 3 times. This experiment will also be finished under 3 different wearing conditions like the basic force experiment above.

\section{Results and Analyses}

In this section, first some of the experiment results will be presented. Then the data analysis methods of the correlation analysis and the regression analysis will be employed to mine the inner relationship among these measurement results. Finally, we will compare the correlation and find the relationship between the basic and integrated experiments above. In the following section, for the sake of simpleness, we define the state of wearing EVA glove under 0kPa as the wearing EVA glove state 1; while we define the state of wearing the EVA glove under $40 \mathrm{kPa}$ as the wearing EVA glove state 2 .

Results of Basic Force Measurement Experiment. Before formal experiment, we test the force results of different operation postures. The experiment results show us that the small difference of the posture does not influence the measurement result prominently. So we choose the posture of turning the front palm to the up direction as the standard operation posture. Some of the experiment results are shown in Fig. 3. In Fig. 3, the blue rectangles are the results of bare hand state; the green rectangles are the results of wearing EVA glove state 1; while the yellow rectangles are the results of wearing EVA glove state 2. The x-axis represents the size of force moment. The y-axis is the size of force. From Fig. 3 we can see that the results of the hold forces in the wearing EVA glove state 1 and 2 are smaller than the results of the hold force under the bare hand state. These results reflect the ergonomics decline of the wearing EVA glove state. On the contrary, the results of screw and pinch forces under wearing EVA glove state 1 is large than those of forces under bare hand state. Since the screw and pinch force measurement devices are small for operation, the subjects would like to exert more forces with the protection of EVA glove.

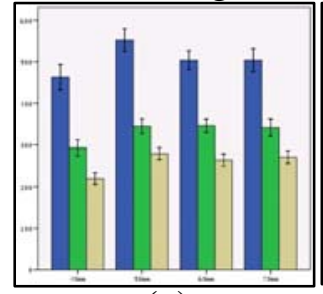

(a)

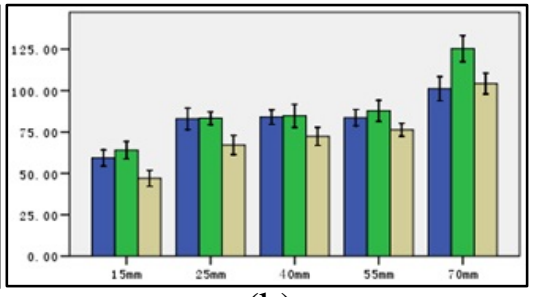

(b)

Figure 3. Records of the

measurement experiment.

basic force

Table2 Correlation Analysis Results of the Hold Force.

\begin{tabular}{|c|c|c|c|c|}
\hline \multicolumn{2}{|c|}{ Correlation Coefficient } & \multicolumn{3}{c|}{ State Type } \\
\cline { 3 - 5 } & Bare Hand State & 1.0 & 0.73 & 0.45 \\
\hline \multirow{3}{*}{ State Type } & EVA Glove State 1 & 0.73 & 1.0 & 0.54 \\
\cline { 2 - 5 } & EVA Glove State 2 & 0.45 & 0.54 & 1.0 \\
\hline
\end{tabular}

Published by Atlantis Press, Paris, France.

(C) the authors 
After the experiment, we can learn that the maximum force will happen when the moments of the force sensor are $50 \mathrm{~mm}, 70 \mathrm{~mm}, 70 \mathrm{~mm}$ for hold force, pinch force and screw force respectively. Table 2 shows us the Pearson correlation results of hold force. We can see among the three types of correlation results, the result of bare hand state also has a correlation with that of the wearing EVA glove state 2. That means we can estimate the force size of the wearing EVA glove under 40kPa by the measurement results of the bare hand state. The similar results can also be found when we calculate the correlation of the other forces. As a result, we can suppose this assumption is reasonable: the data captured in a complex condition may be estimated by the data which are measured under good condition.

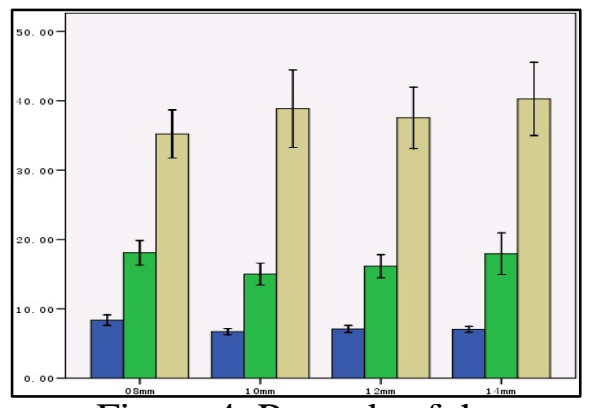

Figure 4. Records of the integrated experiment.

Results of Integrated Force

Measurement Experiment. Fig. 4 shows us the operation time of the integrated force measurement experiments. From Fig. 4 we can see that the operation of the wearing EVA glove state 2 cost the longest time; while the operation of the bare hand state took the shortest time. We also can see the average operation time of these states in Fig. 4. For example when the force moment is $14 \mathrm{~mm}$, the average times of bare hand, wearing EVA glove state 1 and wearing EVA glove state 2 are 7.3s, 18.5s, and 40.2s respectively. During the experiment, we find that all the subjects will spend the similar time to finish the operation task under the same wearing state although their basic forces measurement results are various. From these results we can make an assumption that the operation tasks of our experiment are not too complex for them to finish. So these operations can be selected as some typical operation actions in the space mission in the future.

Correlation Analysis between the Basic and the Integrated Experiments. In Table 3, the correlation results between the forces of the wearing EVA glove state 2 of the basic force experiment and the operation time of the integrated operation experiment is calculated. From these results we can see the hold force and the pinch force have a good correlation with the integrated operation time; while the performance of the screw force is not so good. This result may be explained that the agility of the EVA glove still needs to be improved, especially for the wrist joint. Some subjects complaint the existence of the resistance when they twist their wrist in the process of the experiment. Other subjects reported that they could not nip the force sensor tightly because the clumsiness of the EVA glove.

Table3 Correlation Analysis Results of the Basic and Intergrated Experiments.

\begin{tabular}{|c|c|c|c|}
\hline $\begin{array}{c}\text { Measurement } \\
\text { Index }\end{array}$ & $\begin{array}{c}\text { Parameter Test of } \\
\text { Correlation }\end{array}$ & $\begin{array}{c}\text { Parameter Test of } \\
\text { Non-correlation }\end{array}$ & $\begin{array}{c}\text { Correlation } \\
\text { Coefficient }\end{array}$ \\
\hline Hold Force & 0.002 & 0.026 & -0.391 \\
\hline Pinch Force & 0.003 & 0.006 & -0.394 \\
\hline Screw Force & 0.009 & 0.142 & -0.333 \\
\hline
\end{tabular}

Fig. 5 shows us the regression analysis results between the basic and integrated experiments of the hold and pinch forces. The x-axis of Fig. 5 is the force size of the basic experiment; while the y-axis is the operation time of integrated experiment. The linear regression method, the logarithm regression method and the exponent regression method, etc are all utilized to test the regression characters of our experiment data. From Fig. 5 we can see that the hold force and the pinch force can pass the regression test for most of the regression methods. These results exist extensively in our experiment for other subjects. That means the force data of wearing EVA glove under $40 \mathrm{kPa}$ state and the 
operation time of the integrated experiment have some potential mathematic relationships with each other. So we can build an experiential mathematic model by our experiment method for each astronaut in the future when we want to improve the reliability of the EVA mission.

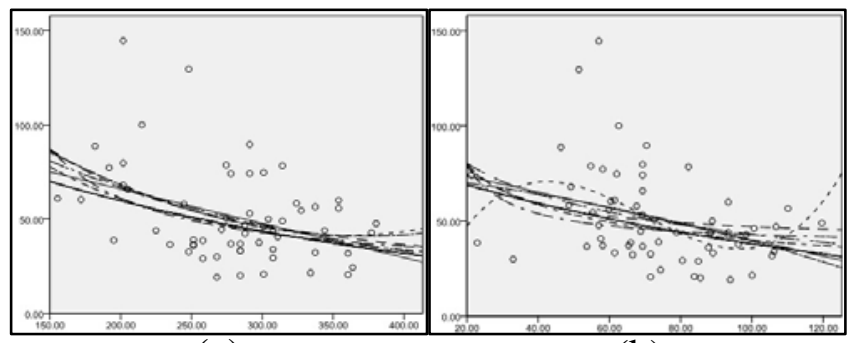

(a)

(b)

Figure 5. Regression analysis between the result of the typical operation forces of the basic force measurement experiment and the result of the operation time of the integrated

\section{Discussions and Suggestions}

Experiment Discussions. From the experiment and analysis results above we can see that 1) the correlation and the regression relationship between the wearing EVA glove state 2 and the bare hand state are prominent for all the 20 subjects in general. The average decline level of force of these two states is about $48 \%$. This result shows that the basic design of the EVA glove is good. No subjects reported the abnormal sense or operation difference of their habit when they finish the experiment. 2) The correlation between the force of the basic force measurement experiment and the operation time of the integrated force measurement experiment is prominent. This result means that the one, whose forces are measured to be small, will take more time to finish the integrated operation tasks. This result also shows the rationality of the ergonomic design of the EVA glove. 3) According to the experiment and analysis results above; we can estimate the force of the wearing EVA glove state 2 by the measurement results of the bare hand state. So we can build a benchmark database of typical operation force for each astronaut to improve the mission security in orbit in the future.

Ergonomics Suggestions. From our research work we can get some ergonomic suggestions for the performance improvement of a certain type of EVA glove. 1) The making material of the EVA glove can be improved. Currently, most parts of the EVA glove are made up of the rubber. So when the pressure in the EVA glove is high, the rigidity of the glove will be increased too. If the material of the EVA glove is softer, the glove will be used more easily. 2) The EVA glove should attach to the skin of the subject's hand well. That means each astronaut should have his own appropriative EVA glove in the future. Only by this means, the shape of EVA glove will accord with the shape of the astronaut's hands well. 3) Some force aid devices [6] can be added on the EVA glove. In this experiment the subjects told us that they felt the difficulties when they tried to crook their fingers or twist their wrist once the glove pressure was high enough. So in the future some force aid device can be installed in these joint of the EVA glove to help the astronaut to finish their operation mission easily.

\section{Conclusions}

In this paper an ergonomic evaluation method is utilized to investigate the force character of a certain type of Extra Vehicular Activity (EVA) glove. 20 male subjects are selected to finish a series of force experiments under different wear states and different pressure levels. By this method, the experiment results show us that the force of bare hand state has a correlation with that of the state of wearing EVA glove under 40Pka pressure. This result can be utilized to analyze the operation force related mission in the space station because they can be estimated by the force result of bare hand state. Besides, these results can guide the design of the improved space suit. In the future, the research of the EVA glove under other situation, such as the task in the neutral buoyancy pool, will be designed. 


\section{Acknowledgement}

This work is supported by the National Basic Research Program of China (973 Program No. 2011CB711000) and the Key Research Program of our Human Factors Engineering Laboratory (No. 2009SY5404003). Thanks Dr. Haoting LIU for his advices on our research work.

\section{References}

[1] R. J. Adams, A. B. Olowin, B. Hannaford, O. S. Sands, in: Tactile Data Entry for Extravehicular Activity, IEEE World Haptics Conference, (2011), p. 305-310.

[2] K. Tanaka, et al., in: Mobility of a Gas-pressurized Elastic Glove for Extravehicular Activity, Acta Astronautica, vol. 66, (2010), p. 1039-1043.

[3] R. R. Bishu, G. Klute, in: Force-endurance Capabilities of Extra-vehicular Activity (EVA) Gloves at Different Pressure Levels, NASA Technical Report, (1993), p. 1-18.

[4] F. A. Korona, D. L. Akin, in: Evaluation of a Hybrid Elastic EVA Glove, Society of Automobile Engineers Technical Paper 2001-01-2311, (2002), p. 1-16.

[5] S. Thompson, M. Mesloh, S. England, E. Benson, S. Raiulu, in: The Effects of Extravehicular Activity (EVA) Glove Pressure on Tactility, Proceedings of the Human Factors and Ergonomics Society Annual Meeting, (2011), p. 1385-1388.

[6] A. Favetto, et al., in: Towards a Hand Exoskeleton for a Smart EVA Glove, IEEE International Conference on Robotics and Biomimetics, (2010), p. 1293-1298. 